\title{
The APOSTEL recommendations for reporting quantitative optical coherence tomography studies OPEN
}

Andrés Cruz-Herranz, MD*

Lisanne J. Balk, $\mathrm{PhD*}$

Timm Oberwahrenbrock, $\mathrm{PhD}$

Shiv Saidha, MD

Elena H. Martinez-

Lapiscina, MD, $\mathrm{PhD}$

Wolf A. Lagreze, MD

Joel S. Schuman, MD

Pablo Villoslada, MD, $\mathrm{PhD}$

Peter Calabresi, MD

Laura Balcer, MD

Axel Petzold, MD, PhD

Ari J. Green, MD, MCR

Friedemann Paul, MD

Alexander U. Brandt, MD*

Philipp Albrecht, MD*

On behalf of the

IMSVISUAL

consortium

Correspondence to

Dr. Albrecht:

phil.albrecht@gmail.com

Supplemental data at Neurology.org

\section{ABSTRACT}

Objective: To develop consensus recommendations for reporting of quantitative optical coherence tomography (OCT) study results.

Methods: A panel of experienced OCT researchers (including 11 neurologists, 2 ophthalmologists, and 2 neuroscientists) discussed requirements for performing and reporting quantitative analyses of retinal morphology and developed a list of initial recommendations based on experience and previous studies. The list of recommendations was subsequently revised during several meetings of the coordinating group.

Results: We provide a 9-point checklist encompassing aspects deemed relevant when reporting quantitative OCT studies. The areas covered are study protocol, acquisition device, acquisition settings, scanning protocol, funduscopic imaging, postacquisition data selection, postacquisition data analysis, recommended nomenclature, and statistical analysis.

Conclusions: The Advised Protocol for OCT Study Terminology and Elements recommendations include core items to standardize and improve quality of reporting in quantitative OCT studies. The recommendations will make reporting of quantitative OCT studies more consistent and in line with existing standards for reporting research in other biomedical areas. The recommendations originated from expert consensus and thus represent Class IV evidence. They will need to be regularly adjusted according to new insights and practices. Neurology ${ }^{\circledR}$ 2016;86:2303-2309

\section{GLOSSARY}

APOSTEL = Advised Protocol for OCT Study Terminology and Elements; GEE = generalized estimating equation models; IMSVISUAL = International Multiple Sclerosis Visual; MS = multiple sclerosis; OCT = optical coherence tomography; ON = optic neuritis.

Optical coherence tomography (OCT) utilizes near infrared light to generate high-resolution cross-sectional images of biological tissue. ${ }^{1}$ Since its development, OCT has been used for the diagnosis and monitoring of numerous primary ocular diseases. With ongoing enhancement of resolution in newer devices and the development of powerful and reliable image processing algorithms, OCT is being increasingly employed to measure the effects of axonal and neuronal damage caused by retinal diseases and optic neuropathies. In recent years, such a quantitative approach has extended the application of OCT to many neurologic disorders with known damage to the visual pathway with multiple sclerosis (MS) and neuromyelitis optica spectrum disorders being the most important. OCT is a sensitive tool for tracking structural changes of the retina,

\footnotetext{
*These authors contributed equally to this work as first/senior authors.

From the Multiple Sclerosis Center (A.C.-H., P.V., A.J.G.), Department of Neurology, University of California San Francisco; Departments of Neurology and Ophthalmology (L.J.B., A.P.), VU University Medical Centre, Amsterdam, the Netherlands; Charité-Universitätsmedizin Berlin (T.O., F.P., A.U.B.), NeuroCure Clinical Research Center; Department of Neurology (T.O., F.P., A.U.B.), Experimental and Clinical Research Center, Max Delbrueck Center for Molecular Medicine, Berlin, Germany; Department of Neurology (S.S., P.C.), Johns Hopkins University, Baltimore, MD; Center of Neuroimmunology and Department of Neurology (E.H.M.-L., P.V.), Institut d'Investigacions Biomèdiques August Pi i Sunyer (IDIBAPS), Hospital Clinic of Barcelona, Spain; Eye Center (W.A.L.), University Medical Center, Freiburg, Germany; Departments of Ophthalmology (J.S.S.) and Neurology (L.B.), New York University School of Medicine, New York; Moorfields Eye Hospital and The National Hospital for Neurology and Neurosurgery (A.P.), London, UK; and Department of Neurology (P.A.), Medical Faculty, Heinrich-Heine University Düsseldorf, Germany.

Coinvestigators are listed on the Neurology ${ }^{\circledR}$ Web site at Neurology.org.

Go to Neurology.org for full disclosures. Funding information and disclosures deemed relevant by the authors, if any, are provided at the end of the article. The Article processing charge was paid by the authors.

This is an open access article distributed under the terms of the Creative Commons Attribution-NonCommercial-NoDerivatives License 4.0 (CC BY-NC-ND), which permits downloading and sharing the work provided it is properly cited. The work cannot be changed in any way or used commercially.
} 
including the macula and optic nerve head, in inflammatory, ${ }^{2-7}$ degenerative, ${ }^{8-12}$ vascular, ${ }^{13,14}$ and metabolic ${ }^{15}$ diseases of the CNS.

AIMS AND USE As the number of quantitative OCT studies in neurology rapidly increases (more than 500 articles reported in PubMed to date) and varying devices and image processing technologies have come into play, there is a need for the development of consistent and coherent standardized reporting recommendations. Harmonious reporting is important for a critical assessment of the strengths and weaknesses of a study. In previous studies, ambiguous reporting has led to uncertainty about different methodologic aspects, such as scan protocols, the use of quality control criteria, and inclusion or exclusion of patients or eyes. The lack of more detailed information on such topics limits the ability to compare data and to apply and generalize findings from these studies. Herein, we present the Advised Protocol for OCT Study Terminology and Elements recommendations (APOSTEL recommendations). They have been developed to outline core information that should be provided when reporting quantitative OCT studies. As such, the recommendations will be instructive for researchers reporting OCT studies that quantitatively assess retinal layer thicknesses and related data. Adhering to these recommendations will improve interstudy interpretability and comparability, ultimately helping to advance research and the clinical application of OCT in the study of neurologic diseases.

The APOSTEL recommendations are designed to complement existing and well-established reporting guidelines ${ }^{16}$ openly available through the equator network (http://www.equator-network.org), but add specific parameters for the reporting of OCT data. We encourage authors to consider the APOSTEL recommendations when quantitative OCT data are to be reported in a study. Likewise, we invite reviewers and journal editors to support adherence to these recommendations when considering OCT studies for publication. In table 1 , we provide a quick and easy-to-use checklist of the APOSTEL recommendations.

DEVELOPMENT The APOSTEL recommendations were conceived during convened meetings of the authors at the 2015 European Academy of Neurology meeting in Berlin, Germany. In these meetings, the aims and scopes of the recommendations were discussed and working groups were established. A preliminary version of the manuscript and the checklist was generated by the working groups, discussed and approved during a joint telephone conference, and then circulated to the members of the International Multiple Sclerosis Visual (IMSVISUAL) consortium (http://www.imsvisual.org) in several rounds for comments and revisions. The members of the consortium approved the final manuscript in person during the 2015 European Committee for Treatment and Research in Multiple Sclerosis (ECTRIMS) convention in Barcelona, Spain, or by e-mail in the case of those who could not attend the meeting.

APOSTEL RECOMMENDATIONS Describe the study protocol. The study design including the inclusion and exclusion criteria and the demographics of the study participants should be described according to established reporting guidelines as may already be applicable to the study, e.g., the Strengthening the Reporting of Observational Studies in Epidemiology (STROBE), Consolidated Standards of Reporting Trials (CONSORT), or Case Reports (CARE) guidelines. ${ }^{16}$ Additional information is required for OCT studies, which generally include information regarding both eyes of each participant. With respect to inclusion and exclusion criteria, authors should define if these were applied at the eye or patient level. In OCT studies, coexisting ocular pathologies represent potential confounders, which can have profound impact on results. Therefore, the patient history and examinations performed to exclude confounding ocular pathologies such as glaucoma or macular degeneration, e.g., funduscopy, tonometry, or slitlamp examinations (for a complete list see reference 17), should be described. We also advise reporting whether patients were tested for refractive errors as well as the cutoff for exclusion based on refraction (typically $\pm 6 \mathrm{D}) .{ }^{18}$ The numbers of eyes and patients excluded should be reported, as well as the criteria leading to such exclusions. In studies comparing OCT examinations to other assessments, e.g., clinical scores or other imaging modalities, it is important to report the time interval between assessments. For OCT studies of neuroinflammatory diseases, reporting the history of and time span from a previous optic neuritis $(\mathrm{ON})$, as well as the number of ON episodes, is of major importance. The authors should clearly define how history of ON was assessed. We strongly recommend the use of standardized definitions of $\mathrm{ON}^{19}$ including information on whether previous $\mathrm{ON}$ diagnoses were arrived at based on clinical, ${ }^{20}$ electrophysiologic, or structural assessments. ${ }^{19}$

State the acquisition device type, name, and version. OCT technology has greatly advanced in recent years. Commercially available OCT devices differ in their optics, data acquisition characteristics, and image analysis algorithms. ${ }^{21}$ It is important to provide detailed information on the devices used (manufacturer, model, interferometric technique), especially in the case of multicenter studies. In addition, detailed information on the software (type, version) used for the acquisition should be provided, if applicable. Wavelength should 
Table 1 Nine-point Advised Protocol for OCT Study Terminology and Elements checklist

Item

1

Study protocol

2

Acquisition device

3

Acquisition Settings

4

Scanning protocol

5

Funduscopic imaging

6

Postacquisition data selection

Postacquisition analysis

8 Nomenclature and abbreviations

$9 \quad$ Statistical approach

\section{Recommendation}

Describe how many OCT operating sites and graders were included

Report the timing of OCT compared to other measurements (same day, delayed)

Describe the inclusion and exclusion criteria

For all OCT devices used, report data on:

Manufacturer

Model

Version

Software version

Clearly describe the settings in which OCT scans were obtained:

Room light conditions

Pupils dilated before examination $(\mathrm{y} / \mathrm{n})$

Number of operators and devices

Clearly describe the scanning protocol, including:

Type of scan (circular, volume, star, line, other)

Location (area of interest, macula, optic nerve head, papillomacular bundle, other?)

Scan parameters (with or without eye tracking)

Volume scan: size of scan area (degrees or millimeters), number of B-scans, alignment of B-scans, number of A-scans per B-scan

Radial scan: size of scan area (degrees or millimeters), number of B-scans, alignment of B-scans, number of A-scans per B-scan

Ring scan: diameter, A-scans/B-scan, manual or automatic placement of ring or method of centering, depth resolution

Line scan: angle, location, number of A-scans, depth resolution

Report other imaging modalities used in addition to OCT (funduscopy, confocal scanning laser ophthalmoscopy, retinal angiography, autofluorescence imaging)

Describe acquisition protocol, including:

Excitation wavelength

Filter sets

Number of frames averaged (if applicable)

Describe image selection process, including:

Quality control criteria (i.e., OSCAR-IB ${ }^{17}$ or other criteria)

Postacquisition discard (number and criteria)

Eye selection strategy (if applicable)

Describe all postacquisition steps:

Software used for processing scans and segmentation (may be different from acquisition software)

Which individual retinal layers were segmented/included

Method of segmentation (automated, semiautomated, or manually)

How potential bias was addressed in the case of manual segmentation (masking)

Grid used for data extraction (size, shape, selected sections)

Define:

Anatomical structures analyzed

Units of provided measurements (e.g., volume or thickness)

Statistical models used for the analyses of OCT data

Abbreviation: OCT $=$ optical coherence tomography. 
be specified, particularly for studies using prototype OCT devices, as different OCT wavelengths may have different imaging characteristics.

Define the acquisition setting. The conditions under which OCT measurements were performed should be standardized and reported. First, the number of individual operators and OCT devices should be reported (sites with more than one operator might describe the intraoperator and interoperator reproducibility of OCT measurements at their sites). Second, there has been some debate over the necessity of pupil dilation. There is concern that pupil dilation might decrease reliability due to higher beam placement variation. ${ }^{22}$ However, the actual effects of pupil dilation have not yet been definitively determined and may in fact be ambiguous. ${ }^{23}$ For example, some patients, especially with severe visual or cognitive impairment, might only be assessable by OCT after pupil dilation. ${ }^{24}$ Handling of pupil dilation should therefore be reported. Finally, several OCT devices offer differing methods for correcting eye movement or position change during acquisition and follow-up measurements. If such a method was used, its application should be reported.

Define OCT scanning protocol. Different OCT scanning protocols influence volumetric imaging and morphometric results. ${ }^{25}$ Often, an OCT imaging session consists of several scan acquisitions per eye. Thus, it is essential to report relevant acquisition parameters of the full measurement protocol, including all scan types employed in a study. The target structure for each scan should be defined and named (e.g., retinal nerve fiber layer, macula, optic nerve). Each scan's shape (e.g., ring, line, volume, radial), orientation (e.g., horizontal, vertical), and size (in degrees or millimeters), as well as other relevant scan-specific parameters, should be reported. For all scans, the authors should provide information on the number of total B-scans, the number of A-scans used for each $\mathrm{B}$-scan, and the number of B-scans averaged.

Define funduscopic imaging. Many OCT devices offer additional fundus imaging modalities such as confocal scanning laser ophthalmoscopy, retinal angiography, and autofluorescence imaging. If included in the analysis, these should be described and the acquisition protocol including the excitation wavelength, filter sets, and the number of frames averaged (if applicable) indicated.

Describe postacquisition data selection. Many OCT image postprocessing algorithms (either included within the device or as external software) have high demands in terms of image contrast and quality. To improve the reliability of results, studies often employ some form of image selection process. A common strategy for increasing image and data quality includes the averaging of several images or obtained values. Likewise, some studies record several images of the same target and subsequently use the optimal image based on defined selection criteria. If such strategies were applied, they should be described in detail, including the selection criteria. In order to ensure a high quality of scans, the use of quality control criteria is recommended. For example, an extensive set of quality control criteria has been published in the form of the OSCAR-IB criteria. ${ }^{17,26}$ Any postacquisition discard should be reported including the number and criteria leading to exclusion of the respective scans. Cutoffs of quantifiable variables such as signal strength should be clearly defined and stated. Numerical values for signal strength are provided in different units and scaling by the different devices so separate cutoffs should be given if more than one device type is used. Depending on the statistical approach (see below), it might be necessary to exclude eyes from the analysis. If this is the case, the strategy of eye selection for statistical analysis should also be reported.

Describe postacquisition data analysis. After acquisition, images have to be further processed and analyzed. Since processing of images is not only device-specific but also dependent on software-implemented algorithms, the name of the software package and its version should be indicated. Authors should report whether the postprocessing performed (e.g., intraretinal layer segmentation) was fully automated, semiautomated with manual correction of obvious errors, or fully manual. In the case of manual postprocessing, authors should report the number of graders, and indicate whether they were masked. Currently, the main postprocessing analysis for retinal OCT data is the generation of volume or thickness data derived from the retinal layers. While data derived from line and ring scans are commonly reported in micrometers, the results derived from volume scans can be reported as mean thickness in $\mu \mathrm{m}$ or volume in $\mathrm{mm}^{3}$. We advise against the use of other units for thickness and volume measurements unless explained in the study objectives. To obtain such measurements, differently sized or shaped areas can be used, such as the ring-shaped grid defined for the Early Treatment of Diabetic Retinopathy Study. ${ }^{27}$ As a matter of fact, values derived from different areas or scans are not necessarily comparable and might also show differences with regard to reliability. ${ }^{28}$ Thus, area shape and size, as well as the source scan used for analysis, should be reported.

Use common nomenclature and abbreviations. The authors should clearly define and describe all structures analyzed. We recommend the nomenclature and abbreviations in table 2 and the figure. Adherence to the nomenclature is especially important when referring 
Table 2 Proposed consensus nomenclature and abbreviations of retinal structures and layers

\begin{tabular}{|c|c|}
\hline Abbreviation & Included structures and layers \\
\hline RNFL & Retinal nerve fiber layer \\
\hline GCL & Ganglion cell layer \\
\hline IPL & Inner plexiform layer \\
\hline INL & Inner nuclear layer \\
\hline OPL & Outer plexiform layer \\
\hline ONL & Outer nuclear layer \\
\hline PRL & $\begin{array}{l}\text { Photoreceptor layer (external limiting membrane to retinal pigment epithelium); } \\
\text { this layer can be separated into the inner segment (IS) and outer segment (OS) }\end{array}$ \\
\hline ISOS & Inner and outer segments junction \\
\hline OPT & Outer photoreceptor tips \\
\hline RPE & Retinal pigment epithelium \\
\hline ILM & Inner limiting membrane \\
\hline ELM & External limiting membrane \\
\hline BM & Bruch membrane \\
\hline ONH & Optic nerve head \\
\hline GCC & Ganglion cell complex (composite of macular RNFL, GCL, and IPL) \\
\hline $\begin{array}{l}\text { GCIP or } \\
\text { GCIPL }\end{array}$ & $\begin{array}{l}\text { Ganglion cell and inner plexiform layer (composite of macular GCL and IPL, } \\
\text { referred to as GCIP or GCIPL in the literature) }\end{array}$ \\
\hline OPNL & Outer plexiform and nuclear layer (composite of OPL and ONL) \\
\hline TRT & $\begin{array}{l}\text { Total retinal thickness (ILM to BM), also named TMT or TMV when referring to the } \\
\text { total macular thickness or volume }\end{array}$ \\
\hline
\end{tabular}

to composite structures. Authors should additionally indicate in which scale structure measurements are provided (e.g., volume or thickness). If authors need to define additional composite structures, the exact definition should be reported.

Define the statistical approach with exact model description. For reporting statistical analyses, authors should adhere to the recommendations in the applicable reporting guidelines. ${ }^{16}$ Regarding the statistical features of OCT studies, the following additional information should be provided. As OCT scans are usually acquired for both eyes in one participant, it is important to describe any strategy applied to account for intereye within-patient dependencies. This latter usually includes either randomly selecting one eye or estimating the mean for non-ON eyes or applying statistical methods able to account for these dependencies, such as general mixed effects models or generalized estimating equation models (GEE). Different strategies might be required for specific questions. ${ }^{29}$ When advanced statistical models are used, authors should report them in sufficient detail, i.e., the model composition and relevant model configurations such as the working correlation matrix in GEE.

IMPLICATIONS AND LIMITATIONS The aim of the APOSTEL recommendations is to provide a consistent basis for assisting authors in composing conclusive reports of studies utilizing quantitative retinal OCT, allowing relevant comparisons across and between studies, and helping readers, reviewers, and editors to evaluate these findings. Furthermore, by emphasizing details that are of importance in such reports, we hope to generally improve the quality of future investigations in this area. Defining the relevant parameters for reporting may prompt researchers to thoroughly consider these criteria during the initial stages of study design. Such an approach could help facilitate OCT research in centers intending to incorporate OCT for clinical trials or other research purposes. Adherence to the recommendations can help avoid common pitfalls in the design and reporting of OCT studies. These recommendations are not intended to impose a rigid format on reports of OCT-based research, but rather to provide suggestions and guidance on which reporting standards to prioritize. As such, the APOSTEL recommendations are intended to complement existing reporting guidelines for clinical studies.

The recommendations should be considered level of evidence IV. They are not based on a systematic review of the literature, but instead rely on the experience of IMSVISUAL's authors and members in conducting, reviewing, and reporting OCT studies. For most of the aspects deemed relevant, formal experimental evidence on their influence on OCT results is lacking, preventing a meta-analytical approach or even a comprehensive review of literature.

The recommendations were not derived from a formalized consensus-building procedure like that of a Delphi process, where anonymized questionnaires are circulated and evaluated in several rounds to achieve consensus. The number of participating individuals was therefore naturally limited and was influenced by existing networks or collaborations of the authors. We acknowledge that this approach can only generate a limited level of evidence (Class IV). However, given the multiple dimensions of the topic and initial heterogeneity of opinions, it was necessary to establish consensus and standardization first. We therefore purposefully chose the term recommendation over guidelines. In order to increase the level of evidence, we plan to refine, evaluate, and validate these recommendations by means of a more formal approach involving a larger forum of researchers to develop more evidence-based guidelines.

Apart from validation, several other concerns need addressing. For example, a limitation of the current recommendations is that they were established by a consortium dominated by neurologists and mainly pertain to the quantitative detection and analysis of retinal changes. Furthermore, the IMSVISUAL consortium is predominantly focused on MS research. 


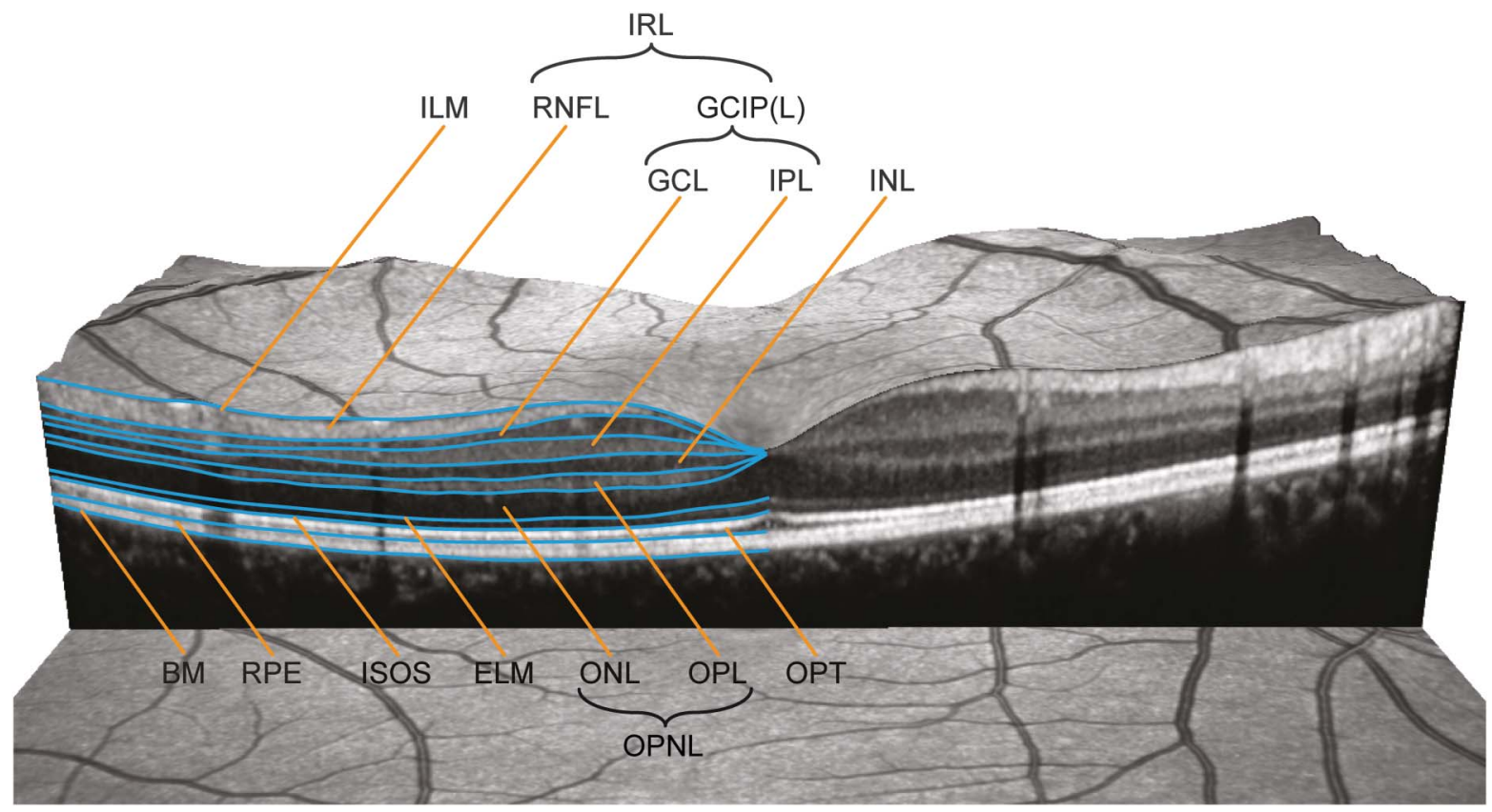

The different layers (and the borders of these) specified in table 2 are illustrated in a central vertical scan through the middle of the foveola. BM = Bruch membrane; $\mathrm{ELM}$ = external limiting membrane; $\mathrm{GCIP}(\mathrm{L})=$ ganglion cell and inner plexiform layer; $\mathrm{GCL}$ = ganglion cell layer; ILM = inner limiting membrane; $\mathrm{INL}=$ inner nuclear layer; IPL = inner plexiform layer; IRL = inner retinal layer; ISOS $=$ inner and outer segments; ONL = outer nuclear layer; OPNL = outer plexiform and nuclear layer; $\mathrm{OPL}=$ outer plexiform layer; $\mathrm{OPT}=$ outer photoreceptor tips; RNFL = retinal nerve fiber layer; RPE = retinal pigment epithelium.

However, several of the authors are involved in OCT research well beyond $\mathrm{MS}^{10,12-15,30}$ and the recommendations have been designed to also apply to studies on patients with other neurologic or ophthalmologic conditions that are increasingly being performed with similar methodology. Further development incorporating these potentially different points of view will be crucial for the broader application and success of the recommendations.

We therefore consider the APOSTEL recommendations as a preliminary but urgently needed step towards further improving the overall quality and utility of quantitative OCT research in neurologic disorders. These recommendations constitute an expert consensus statement and their value will be assessed in the future through use in studies related to visual outcomes in ocular and neurologic disease. The recommendations will require ongoing refinement to increase the level of evidence and to accommodate the continuous evolution of OCT technology. We will update these recommendations in the future, taking into account user comments, criticism, new evidence, and experience. We therefore welcome suggestions for improvement and further development of the criteria, as well as additional areas of application, and invite readers to submit their comments via the IMSVISUAL Web site. Recommendation updates and current checklist versions will be available on the IMSVISUAL Web site (www.imsvisual.org).

\section{AUTHOR CONTRIBUTIONS}

Andrés Cruz-Herranz: study concept and design, acquisition of data, analysis and interpretation, critical revision of the manuscript for important intellectual content. Lisanne J. Balk: study concept and design, acquisition of data, analysis and interpretation, critical revision of the manuscript for important intellectual content. Timm Oberwahrenbrock: analysis and interpretation, critical revision of the manuscript for important intellectual content. Shiv Saidha: analysis and interpretation, critical revision of the manuscript for important intellectual content. Elena $\mathrm{H}$. Martinez-Lapiscina: analysis and interpretation, critical revision of the manuscript for important intellectual content. Wolf A. Lagreze: analysis and interpretation, critical revision of the manuscript for important intellectual content. Joel S. Schuman: analysis and interpretation, critical revision of the manuscript for important intellectual content. Pablo Villoslada: analysis and interpretation, critical revision of the manuscript for important intellectual content. Peter Calabresi: analysis and interpretation, critical revision of the manuscript for important intellectual content. Laura Balcer: analysis and interpretation, critical revision of the manuscript for important intellectual content. Axel Petzold: analysis and interpretation, critical revision of the manuscript for important intellectual content. Ari J. Green: analysis and interpretation, critical revision of the manuscript for important intellectual content. Friedemann Paul: analysis and interpretation, critical revision of the manuscript for important intellectual content. Alexander U. Brandt: analysis and interpretation, study concept and design, acquisition of data, critical revision of the manuscript for important intellectual content, study supervision. Philipp Albrecht: analysis and interpretation, study concept and design, acquisition of data, critical revision of the manuscript for important intellectual content, study supervision.

\section{ACKNOWLEDGMENT}

The authors thank IMSVISUAL and its members for their input into the development of these reporting guidelines.

\section{STUDY FUNDING}

No targeted funding reported. 


\section{DISCLOSURE}

The authors report no disclosures relevant to the manuscript. Go to Neurology.org for full disclosures.

Received December 21, 2015. Accepted in final form March 14, 2016.

\section{REFERENCES}

1. Huang D, Swanson EA, Lin CP, et al. Optical coherence tomography. Science 1991;254:1178-1181.

2. Balk L, Tewarie P, Killestein J, Polman C, Uitdehaag B, Petzold A. Disease course heterogeneity and OCT in multiple sclerosis. Mult Scler 2014;20:1198-1206.

3. Oberwahrenbrock $T$, Ringelstein $M$, Jentschke $S$, et al. Retinal ganglion cell and inner plexiform layer thinning in clinically isolated syndrome. Mult Scler 2013;19: 1887-1895.

4. Saidha S, Syc SB, Ibrahim MA, et al. Primary retinal pathology in multiple sclerosis as detected by optical coherence tomography. Brain 2011;134:518-533.

5. Schneider E, Zimmermann H, Oberwahrenbrock T, et al. Optical coherence tomography reveals distinct patterns of retinal damage in neuromyelitis optica and multiple sclerosis. PLoS One 2013;8:e66151.

6. Outteryck O, Majed B, Defoort-Dhellemmes S, Vermersch P, Zéphir H. A comparative optical coherence tomography study in neuromyelitis optica spectrum disorder and multiple sclerosis. Mult Scler 2015;21:1781-1793.

7. Bennett JL, de Seze J, Lana-Peixoto M, et al. Neuromyelitis optica and multiple sclerosis: Seeing differences through optical coherence tomography. Mult Scler 2015; 21:678-688.

8. Cheung CY, Ong YT, Hilal S, et al. Retinal ganglion cell analysis using high-definition optical coherence tomography in patients with mild cognitive impairment and Alzheimer's disease. J Alzheimers Dis 2015;45:45-56.

9. Bodis-Wollner I, Miri S, Glazman S. Venturing into the no-man's land of the retina in Parkinson's disease. Mov Disord 2014;29:15-22.

10. Roth NM, Saidha S, Zimmermann H, et al. Photoreceptor layer thinning in idiopathic Parkinson's disease. Mov Disord 2014;29:1163-1170.

11. Stricker S, Oberwahrenbrock T, Zimmermann H, et al. Temporal retinal nerve fiber loss in patients with spinocerebellar ataxia type 1. PLoS One 2011;6:e23024.

12. Ringelstein M, Albrecht P, Südmeyer M, et al. Subtle retinal pathology in amyotrophic lateral sclerosis. Ann Clin Transl Neurol 2014;1:290-297.

13. Albrecht P, Blasberg C, Lukas S, et al. Retinal pathology in idiopathic moyamoya angiopathy detected by optical coherence tomography. Neurology 2015;85:521-527.

14. Ringelstein M, Albrecht P, Kleffner I, et al. Retinal pathology in Susac syndrome detected by spectral-domain optical coherence tomography. Neurology 2015;85:610-618.

15. Albrecht P, Müller AK, Ringelstein M, et al. Retinal neurodegeneration in Wilson's disease revealed by spectral domain optical coherence tomography. PLoS One 2012; 7:e49825.

16. The EQUATOR network. Enhancing the Quality and Transparency of Health Research. Available at: http:// www.equator-network.org/. Accessed February 17, 2016.

17. Tewarie P, Balk L, Costello F, et al. The OSCAR-IB consensus criteria for retinal OCT quality assessment. PLoS One 2012;7:e34823.

18. Leung CKS, Mohamed S, Leung KS, et al. Retinal nerve fiber layer measurements in myopia: an optical coherence tomography study. Invest Ophthalmol Vis Sci 2006;47: 5171-5176.

19. Petzold A, Wattjes MP, Costello F, et al. The investigation of acute optic neuritis: a review and proposed protocol. Nat Rev Neurol 2014;10:447-458.

20. Beck RW, Cleary PA, Anderson MM, et al. A randomized, controlled trial of corticosteroids in the treatment of acute optic neuritis: The Optic Neuritis Study Group. N Engl J Med 1992;326:581-588.

21. Warner CV, Syc SB, Stankiewicz AM, et al. The impact of utilizing different optical coherence tomography devices for clinical purposes and in multiple sclerosis trials. PLoS One 2011;6:e22947.

22. Balk LJ, de Vries-Knoppert WA, Petzold A. A simple sign for recognizing off-axis OCT measurement beam placement in the context of multicentre studies. PLoS One 2012;7:e48222

23. Alizadeh Y, Panjtanpanah MR, Mohammadi MJ, Behboudi H, Kazemnezhad Leili E. Reproducibility of optical coherence tomography retinal nerve fiber layer thickness measurements before and after pupil dilation. J Ophthalmic Vis Res 2014;9:38-43.

24. Smith M, Frost A, Graham CM, Shaw S. Effect of pupillary dilatation on glaucoma assessments using optical coherence tomography. Br J Ophthalmol 2007; 91:1686-1690

25. Domalpally A, Danis RP, Zhang B, Myers D, Kruse CN. Quality issues in interpretation of optical coherence tomograms in macular diseases. Retina 2009;29:775-781.

26. Schippling S, Balk L, Costello F, et al. Quality control for retinal OCT in multiple sclerosis: validation of the OSCAR-IB criteria. Mult Scler 2015;21:163-170.

27. Early Treatment Diabetic Retinopathy Study Research Group. Photocoagulation for diabetic macular edema: Early Treatment Diabetic Retinopathy study report number 1. Arch Ophthalmol 1985;103:1796-1806.

28. Oberwahrenbrock T, Weinhold M, Mikolajczak J, et al. Reliability of intra-retinal layer thickness estimates. PLoS One 2015;10:e0137316.

29. Fan Q, Teo Y-Y, Saw S-M. Application of advanced Statistics in ophthalmology. Invest Ophthalmol Vis Sci 2011;52:6059-6065.

30. Albrecht P, Müller A-K, Südmeyer M, et al. Optical coherence tomography in parkinsonian syndromes. PLoS One 2012;7:e34891. 


\section{Neurology}

\section{The APOSTEL recommendations for reporting quantitative optical coherence tomography studies}

Andrés Cruz-Herranz, Lisanne J. Balk, Timm Oberwahrenbrock, et al. Neurology 2016;86;2303-2309 Published Online before print May 25, 2016

DOI 10.1212/WNL.0000000000002774

This information is current as of May 25, 2016

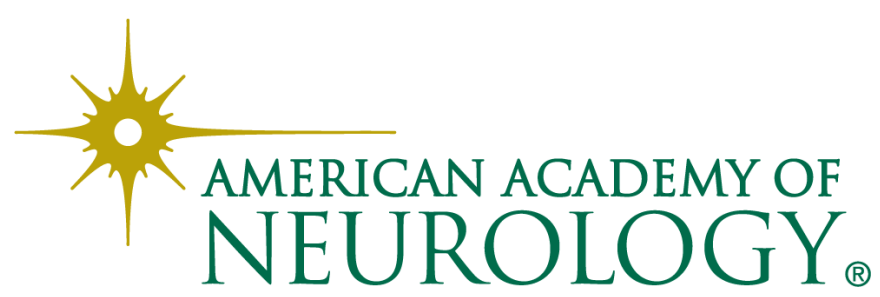




\section{Updated Information \& Services}

\section{Supplementary Material}

References

Citations

Subspecialty Collections

Permissions \& Licensing

Reprints including high resolution figures, can be found at: http://n.neurology.org/content/86/24/2303.full

Supplementary material can be found at: http://n.neurology.org/content/suppl/2016/05/25/WNL.0000000000002 774.DC1

This article cites 29 articles, 6 of which you can access for free at: http://n.neurology.org/content/86/24/2303.full\#ref-list-1

This article has been cited by 14 HighWire-hosted articles: http://n.neurology.org/content/86/24/2303.full\#\#otherarticles

This article, along with others on similar topics, appears in the following collection(s):

\section{All Imaging}

http://n.neurology.org/cgi/collection/all_imaging

All Neuro-ophthalmology

http://n.neurology.org/cgi/collection/all_neuroophthalmology

Clinical trials Methodology/study design

http://n.neurology.org/cgi/collection/clinical_trials_methodology_study _design_

\section{Retina}

http://n.neurology.org/cgi/collection/retina

Information about reproducing this article in parts (figures,tables) or in its entirety can be found online at:

http://www.neurology.org/about/about_the_journal\#permissions

Information about ordering reprints can be found online:

http://n.neurology.org/subscribers/advertise

Neurology ${ }^{\circledR}$ is the official journal of the American Academy of Neurology. Published continuously since 1951, it is now a weekly with 48 issues per year. Copyright @ 2016 American Academy of Neurology. All rights reserved. Print ISSN: 0028-3878. Online ISSN: 1526-632X.

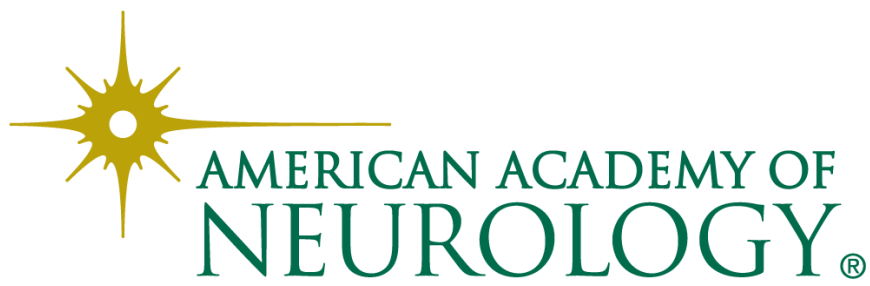

